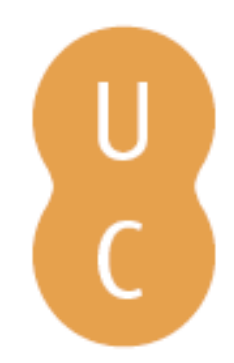

\title{
nombalina
}

\section{Modelação e análise de perdas associadas ao risco sísmico}

Autor(es): $\quad$ Sá, Luis; Pires, Patrícia; Henriques, Paulo; Telhado, Maria João

Publicado por: Imprensa da Universidade de Coimbra; RISCOS - Associação

URL

persistente: URI:http://hdl.handle.net/10316.2/34820

DOI: $\quad$ DOI:http://dx.doi.org/10.14195/978-989-96253-3-4_29

Accessed : $\quad$ 26-Apr-2023 15:07:02

A navegação consulta e descarregamento dos títulos inseridos nas Bibliotecas Digitais UC Digitalis, UC Pombalina e UC Impactum, pressupõem a aceitação plena e sem reservas dos Termos e Condições de Uso destas Bibliotecas Digitais, disponíveis em https://digitalis.uc.pt/pt-pt/termos.

Conforme exposto nos referidos Termos e Condições de Uso, o descarregamento de títulos de acesso restrito requer uma licença válida de autorização devendo o utilizador aceder ao(s) documento(s) a partir de um endereço de IP da instituição detentora da supramencionada licença.

Ao utilizador é apenas permitido o descarregamento para uso pessoal, pelo que o emprego do(s) título(s) descarregado(s) para outro fim, designadamente comercial, carece de autorização do respetivo autor ou editor da obra.

Na medida em que todas as obras da UC Digitalis se encontram protegidas pelo Código do Direito de Autor e Direitos Conexos e demais legislação aplicável, toda a cópia, parcial ou total, deste documento, nos casos em que é legalmente admitida, deverá conter ou fazer-se acompanhar por este aviso.

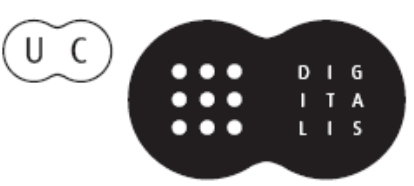



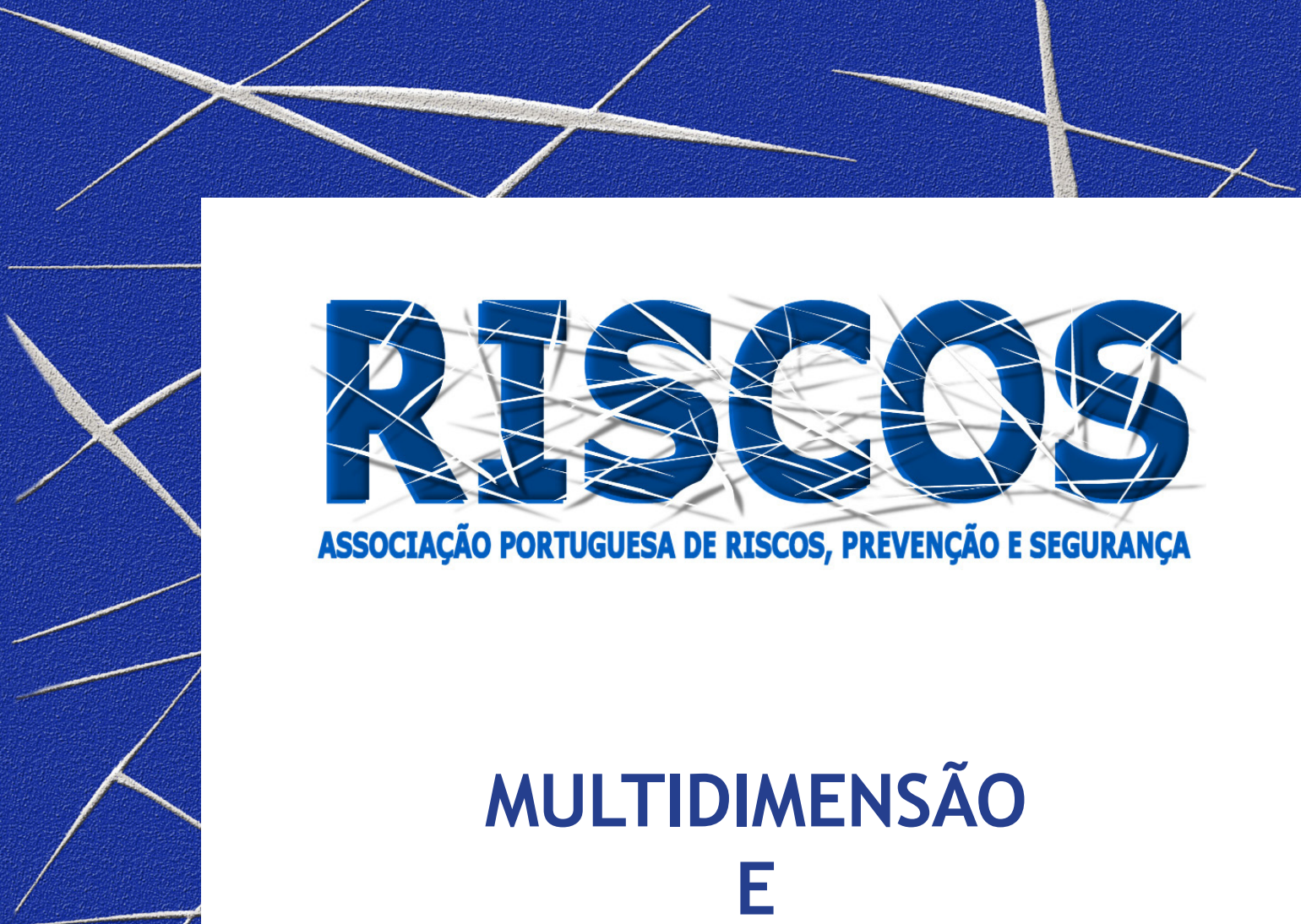

ASSOCIAÇÃO PORTUGUESA DE RISCOS, PREVENCCÃO E SEGURANÇA

MULTIDIMENSÃO

E
TERRITÓRIOS DE RISCO

III Congresso Internacional

I Simpósio Ibero-Americano

VIII Encontro Nacional de Riscos

Guimarães

2014 


\title{
MODELAÇÃO E ANÁLISE DE PERDAS ASSOCIADAS AO RISCO SÍSMICO
}

\author{
Luis Sá \\ Autoridade Nacional de Proteção Civil \\ luis.sa@prociv.pt \\ Patrícia Pires \\ Autoridade Nacional de Proteção Civil \\ patricia.pires@prociv.pt \\ Paulo Henriques \\ Câmara Municipal de Lisboa \\ paulo.franco@cm-lisboa.pt \\ Maria João Telhado \\ Câmara Municipal de Lisboa \\ joão.telhado@cm-lisboa.pt
}

\begin{abstract}
RESUMO
Portugal possui um elevado risco sísmico tendo em conta as vulnerabilidades associadas à ocupação humana bem como ao histórico de eventos sísmicos registados. Face à inevitabilidade da ocorrência de sismos, a abordagem a esta questão num contexto nacional deve assentar na análise dos seus aspetos fundamentais, $\mathrm{e}$ que simultaneamente reflitam necessariamente sobre os fatores que contribuem para a existência de um risco "real" de desastre para a sociedade. É inestimável a existência e a manutenção de um simulador de danos resultantes de um sismo com a capacidade de visualização de um cenário, com indicação de danos potenciais, cuja tipologia possui uma incerteza difícil de determinar empiricamente. Presentemente existem duas regiões do país abrangidas por tais ferramentas, Lisboa e o Algarve, persistindo uma lacuna no que respeita ao restante território. Assim, numa tentativa para colmatar este facto, a Autoridade Nacional de Proteção Civil (ANPC) e a Câmara Municipal de Lisboa (CML) através do seu Serviço Municipal de Proteção Civil, unindo esforços e conhecimento técnico-científico, estão a desenvolver um protótipo de simulador de Risco Sísmico cuja metodologia poderá ser aplicável ao continente português de forma transversal.
\end{abstract}

Palavras-chave: Modelação. Risco Sísmico. Consequências

Painel: Riscos Geológicos

\section{Introdução}

É inestimável a existência e a manutenção de um simulador de danos resultantes de um sismo com a capacidade de visualização de um cenário, com indicação de danos potenciais, cuja tipologia possui incerteza difícil de determinar empiricamente. Até agora só existiam duas regiões do país abrangidas por tais ferramentas, Lisboa e o Algarve, persistindo uma lacuna no que respeita ao restante território. Assim, numa tentativa de colmatar esta realidade, a ANPC e a CML, unindo esforços e conhecimento técnico, estão a desenvolver um protótipo de simulador cuja metodologia poderá ser aplicável ao continente português de forma transversal. No presente trabalho, dá-se a conhecer as etapas desenvolvidas na construção do protótipo do simulador:

I. Recolher, tratar, compatibilizar e integrar no simulador dados e metodologias nacionais e internacionais desenvolvidas durante as ultimas décadas na área do risco sísmico que contaram com a participação da ANPC e da Proteção Civil do município de Lisboa.

II. Modelar o simulador com os principais modelos de cálculo nas seguintes áreas de desenvolvimento, i) leis de Atenuação, modelo de cálculo dos efeitos dos solos na propagação da onda sísmica desde o substrato rochoso até superfície e quantificação da ação sísmica à 
superfície; ii) distribuição espacial, por subsecção estatística, da população móvel e a população presente "in-house" e "out-house" para cada mês do ano e ao longo de vários períodos do dia; iii) modelos de cálculo de danos para o Edificado, População e para a componente financeira e iv) resultados da modelação de Tsunamis, sob a forma de Altura de Onda,

III. Conceber, desenvolver e adaptar o simulador para utilização pela ANPC e posteriormente noutros serviços de proteção civil distrital e municipal, como é o caso de Lisboa.

\section{Desenvolvimento de Conteúdos}

Como pré-requisitos, foram utilizadas como fonte de informação, e de dados, diversas variáveis estudadas para a realização do Estudo do Riscos Sísmico da Área Metropolitana de Lisboa $e$ Concelhos Limítrofes (AML-CL) e do Estudo do Risco sísmico e Tsunamis do Algarve (ERSTA). Através da colaboração conjunta com o SMPC do município de Lisboa (CML), obtiveram-se informações técnicas sobre o simulador municipal de risco sísmico desenvolvido para esta a cidade na década de 90 , do qual derivaram metodologicamente os actuais simuladores em uso pela ANPC, com a vantagem de recorrer ao uso de compilações em código aberto. Outras fontes de informação que merecem destaque são as informações técnicas apensas ao software HAZUS/ FEMA e ao projeto de investigação EU FP-6 - NERIES. Desenvolvido sobre o ambiente EXCEL, em linguagem Visual Basic, todos os scripts presentes neste simulador são editáveis por um utilizador credenciado, a ser definido consoante o tipo de perfil do utilizador. Somente como ferramenta de visualização optou-se pelo já decano ArcView 3.2 da ESRI por ainda ser um software de utilização rápida, eficaz e ainda atualmente reconhecido como tal pela maioria dos utilizadores de SIG, correndo em ambientes tão distintos como o antigo Windows 98 ou o atual Windows 8 , o que denota a sua abrangente versatilidade. A construção da metodologia assenta na premissa que o risco sísmico é estimado em função do perigo de ocorrência de um fenómeno e da exposição dos elementos vulneráveis a essa ocorrência.

Leis de Atenuação - Esta análise foi baseada em sete leis de atenuação criadas com dados de diferentes áreas do globo, incluindo a Península Ibérica a nível micro e ao nível macro. Através de uma metodologia iterativa assente numa combinação dessas sete leis de atenuação, obtiveram-se resultados que integram uma relação satisfatória entre magnitude, epicentro e a distância a este.

Impedância - A modificação do movimento sísmico devido às condições topográficas e geotécnicas locais é chamada de efeito local. Esta amplificação, ou atenuação é obtida comparando a resposta de um sítio com a de um local de referência, isto é, um ponto localizado na rocha plana. $O$ contraste de impedância entre sedimentos e rocha caracteriza as propriedades de um solo. Assim, quanto menor o contraste de impedância, mais brandos os sedimentos (na figura identificado com a letra A) são quando comparados com o substrato rochoso/rocha-mãe (B). Considera-se o caso simples de uma camada de solo horizontal ao longo de um espaço elástico. A partir da rasterização da carta geológica de Portugal 1:1.000.000 e do cruzamento da informação associada com as subsecções estatísticas do INE de 2011, via geoprocessamento em SIG, foi possível obter qual a formação geológica predominante por subsecção. Seguidamente obtiveram-se para cada formação geológica os valores de referência para a velocidade de propagação de onda e para a densidade específica, tendo-se estabelecido como rocha-mãe os 
Bakun and Wentworth (1997): California, USA

$$
I_{M M I}=3.67+1.17 M_{W}-3.19 \log \left(R_{\text {epi }}\right)
$$

ii. Bakun and Scotti (2006): French SCR

$$
I_{M S K}=4.48+1.27 M_{W}-3.37 \log \left(\sqrt{R_{e p i}{ }^{2}+h^{2}}\right)
$$

iii.

Bakun (2006): Basin \& Range, USA

$$
\begin{aligned}
& I_{\operatorname{mag}}=0.44+1.70 M_{W}-0.0048 \Delta_{h}-2.73 \log \left(\Delta_{h}\right) \\
& \text { where: }
\end{aligned}
$$

$$
\begin{aligned}
& \Delta_{h}=\sqrt{R_{\sigma \mathrm{pi}}^{2}+h^{2}} \\
& h=10 \\
& \sigma=0.58
\end{aligned}
$$

iv. Pasolini et al. (2008): Italy

$$
\begin{aligned}
& I_{M C S}=I_{E}-0.0086\left(\sqrt{R_{C \varphi}{ }^{2}+h^{2}}-h\right)-1.037\left[\ln \left(\sqrt{R_{\varphi \varphi i}{ }^{2}+h^{2}}\right)-\ln (h)\right] \\
& \text { where: } \\
& I_{E}=2.460 M_{W}-5.862 \\
& h=3.91 \\
& \sigma=0.69
\end{aligned}
$$

v.

Esteva \& Rosenblueth (1967): California

$$
\text { IMm }=\mathrm{c} 1+\mathrm{c} 2 \mathrm{M}-\mathrm{c} 3 \ln R
$$

ท.

Lopez et al. (2000): Iberia

$$
I=5.557+0.902 \cdot I_{0}+0.014 \cdot I_{0}^{2}
$$

vii.

Crespellani et al. (1993): Italy

$$
I_{M M}=6.39+1.756 \mathrm{M}-2.747 \ln (R+7)
$$

Figura 1 - Leis de Atenuação usadas

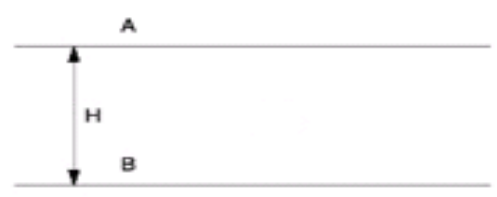

Figura 2 - Modelo Unidimensional de Estratificação 
valores associados às formações do tipo rocha ígnea plutónica ou intrusiva, como são o caso de Monchique, Sintra, Estrela e outras formações rochosas situadas mais a Norte. A partir da informação obtida foi então possível obter uma classificação continental de 19 classes de contraste impedância (B). Estimação da Vulnerabilidade - A vulnerabilidade é definida como nível de danos de um elemento, ou conjunto de elementos expostos, resultante da ocorrência de um fenómeno natural ou tecnológico (Julião et al, 2009), Funções de vulnerabilidade (ou curvas de fragilidade) de um elemento exposto representam a probabilidade de que a sua resposta ao terremoto excede os seus vários estados limites de desempenho com base em considerações físicas e económicas e sociais. Os procedimentos genéricos usados para o desenvolvimento de funções de fragilidade podem ser agrupadas como empíricos ou analíticos. Um exemplo marcante é o proposto por Giovinazzi e Lagomarsino (2004) que levaram à definição de funções de probabilidade de dano com base na escala EMS-98 (Grünthal, 1998). Curvas de Fragilidade - A escala EMS-98 define descrições qualitativas de "poucos", "muitos" e "a maioria" para cinco graus de danos (D1 a D5) para os níveis de intensidade que variam de $\mathrm{V}$ a XII, por cinco ou seis classes diferentes de vulnerabilidade (A a E ou A a F). As matrizes de probabilidade de danos produzidas para cada classe de vulnerabilidade foram relacionadas com o parque edificado através da utilização de um índice de vulnerabilidade empírico que depende do edifício, tipologia e as práticas de construção tipicamente utilizadas. As informações sobre o edificado nacional foram recolhidas a partir dos resultados dos Censos 2011. Um modo para associar cada edifício a uma tipologia foi feito com a ajuda do EMS98. Levando em consideração Teves-Costa et al. (2011), valores mínimos, médios e máximos de índices de vulnerabilidade foram escolhidos por tipologia.

\begin{tabular}{|c|c|c|c|c|c|}
\hline $\begin{array}{c}\text { Date of } \\
\text { consiruction }\end{array}$ & $\begin{array}{c}\text { Number of } \\
\text { floors }\end{array}$ & $\begin{array}{c}\text { Concrete } \\
\text { (EBAR) }\end{array}$ & $\begin{array}{c}\text { Masonny } \\
\text { (EARG) }\end{array}$ & $\begin{array}{c}\text { Adobe/ Nud } \\
\text { (EPAT) }\end{array}$ & $\begin{array}{c}\text { Other } \\
\text { (EORE) }\end{array}$ \\
\hline Before 1919 & All & -- & B & A & A \\
\hline $1920-1960$ & All & C & C & A & A \\
\hline $1961-1985$ & All & D & C & A & A \\
\hline $1986-2009$ & All & E & D & A & A \\
\hline
\end{tabular}

Figura 3 - Tipologia de Edificado (A a E)

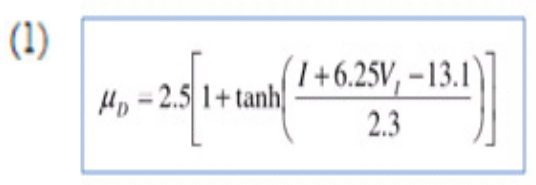

A partir do cruzamento das últimas duas tabelas, obtém-se um valor médio de vulnerabilidade (Vi) para cada classe. Neste ponto, aplica-se a expressão (1) para obter $\mu \mathrm{D}$ que é o nível de vulnerabilidade. Seguidamente a função dano é estimada, usando uma distribuição beta de execução compatível com o software Excel2007 e o MiniTab15. As curvas de fragilidade podem finalmente ser estimadas após o cálculo da função de densidade de probabilidade. Concomitantemente no cálculo da vulnerabilidade utilizado neste simulador recorreu-se simultaneamente ao método Cambridge que, no caso dos edifícios efetivamente colapsados 
(colapso total), considera a taxa de mortalidade de $90 \%$, e a taxa de feridos igual a $10 \%$, seguindo uma distribuição análoga à preconizada no modelo original. Coburn e Spencer (2002) também propuseram um modelo global de estimativa de vítimas, considerando no momento do sismo, por edifício: o tipo de construção, o número de ocupantes descriminando os que se encontram presos no desabamento, a sua ocupação, o número de ocupantes presos, distribuição das lesões em situações de colapso e pós mortalidade em colapso. Estimação do Risco - O valor final de risco será calculado pela combinação do perigo (cálculo das intensidades macrossísmicas via leis de atenuação) com a vulnerabilidade do edificado (através das metodologias de Coburn\&Spencer + Giovinazzi\&Lagomarsino\&Cambridge) expressa em função de danos, i) $n^{\circ}$ de vitimas mortais; $n^{\circ}$ de vítimas com ferimentos ligeiros; $n^{\circ}$ de pessoas desalojadas; ii) $n^{\circ}$ edifícios colapsados; $n^{\circ}$ edifícios com danos severos; $n^{\circ}$ edifícios com danos moderados; $n^{\circ}$ edifícios com danos ligeiros; iii) perdas financeiras e iv) altura de potencial tsunami, em caso de cenário costeiro via Gusyakov e Chubarov (1987).

\section{Conclusão}

Procurou-se com este trabalho proporcionar à ANPC um instrumento de planeamento de emergência e de apoio às políticas de Prevenção e Resposta a situações de ocorrência de sismos de moderada a elevada intensidade. Embora aparentemente complexo, o simulador desenvolvido está desenhado por forma a, sem intervenção do utilizador, definir qualquer cenário, ativando um conjunto de opções pré-definidas, que constituem uma aproximação equilibrada a qualquer situação. Assim, o utilizador apenas terá que fazer opções de formulações alternativas se assim o desejar e quando se sentir preparado para o fazer. Por fim, a introdução de um procedimento que gera de forma automática ficheiros de resultados estatísticos pré-formatados para cada um dos cenários escolhidos, constitui uma forma de, com extrema simplicidade, incorporar toda essa informação em textos, relatórios, mapas e apresentações ou outras formas de comunicação, estudo ou divulgação da informação produzida de apoio à prevenção e à emergência. Esperamos assim ter contribuído para uma mitigação do risco sísmico e um incremento da segurança de quem amamos.

\section{Bibliografia}

Baptista, M. A., Miranda, J. M., Batlo, J., \& Ferreira, H. (2012, April). Tsunami flooding along Tagus estuary, Portugal, the 1531 event. In EGU G.A. Conference Abstracts (Vol. 14, p. 4910).

Bernardini, A., Giovinazzi, S., Lagomarsino, S., \& Parodi, S. (2007). The vulnerability assessment of current buildings by a macroseismic approach derived from the EMS-98 scale.

Coburn, A. Spence, R. (2002) Earthquake Protection, 2nd Edition ISBN: 978-0-470-84923-1

Erdik, M., Sesetyan, K., Demircioglu, M., Hancilar, U., Zulfikar, C., Cakti, E. \& Harmandar, E. (2010). Rapid earthquake hazard and loss assessment for Euro-Mediterranean region. Acta Geophysica, 58(5), 855 892.

Giovinazzi, S., Lagomarsino, S. (2004). A macroseismic method for the vulnerability assessment of buildings. In 13th WCEE, Vancouver, BC, Canada (pp. 1-6).

Gusyakov, V. K., and L. B. Chubarov (1987), Numerical modeling of generation and propagation of tsunami in coastal zone, Izv. Phys. Solid Earth, 23, (11), 53.

Grünthal, G. (ed.): European Macroseismic Scale 1998 (EMS-98). Cahiers du Centre Européen de Géodynamique et de Séismologie 15, Luxembourg, 99 pp., 1998. 


\section{CAPÍTULO 3.1: RISCOS GEOLÓGICOS E GEOMORFOLÓGICOS}

Julião, et al (2009) . Guia metodológico para a produção de cartografia municipal de risco e para a criação de sistemas de informação geográfica (SIG) de base municipal. Autoridade Nacional de Proteção Civil, pp 93.

Oliveira, C. S., Pais, I. (1995). Technical approaches for earthquake emergency planning: Recent application to the city of Lisbon.. London E and FN Spon, 58-72.

Sousa, M.L., (2007). "Hierarquização das regiões de Portugal Continental em função do seu risco sísmico". Sísmica 2007 - $7^{\circ}$ Congresso de Sismologia e Engenharia Sísmica, 26-28 de Setembro, FEUP, Porto, Portugal.

Teves-Costa, P, Barreira E, Omira R (2011). Estimativa de cenários de danos para a cidade de Lisboa. RISK-UE. www.risk-ue.net 